\title{
Developing a New, National Approach to Surveillance for Ventilator-Associated Events: Executive Summary
}

\author{
Shelley S Magill MD PhD ${ }^{1}$, Michael Klompas MD MPH ${ }^{2,3,4}$, Robert Balk $\mathrm{MD}^{5,6}$, \\ Suzanne M Burns RN ACNP MSN RRT ${ }^{6,7}$, Clifford S Deutschman MS MD ${ }^{6,8}$, \\ Daniel Diekema MD ${ }^{9,10}$, Scott Fridkin MD ${ }^{1}$, Linda Greene RN MPS ${ }^{11,12}$, Alice Guh MD MPH ${ }^{1}$, \\ David Gutterman MD ${ }^{6,13}$, Beth Hammer RN MSN ANP-BC ${ }^{6,14}$, David Henderson MD ${ }^{15}$, \\ Dean R Hess PhD RRT ${ }^{16,17,18}$, Nicholas S Hill MD ${ }^{6,19}$, Teresa Horan MPH ${ }^{1}$, Marin Kollef MD ${ }^{6,20}$, \\ Mitchell Levy MD ${ }^{6,21}$, Edward Septimus MD ${ }^{22,23}$, Carole VanAntwerpen RN BSN ${ }^{24,25}$, \\ Don Wright MD MPH ${ }^{26}$, and Pamela Lipsett MD MHPE ${ }^{6,27}$
}

Key words: ventilator-associated pneumonia; ICU; epidemiology; public health; critical care; mechanicalventilation. [Respir Care 2013;58(11):1985-1989]

In September 2011, the Centers for Disease Control and Prevention (CDC) convened a Ventilator-Associated Pneumonia (VAP) Surveillance Definition Working Group to organize a formal process for leaders and experts of key stakeholder organizations to discuss the challenges of VAP

\footnotetext{
1. Division of Healthcare Quality Promotion, Centers for Disease Control and Prevention, Atlanta, GA. 2. Department of Population Medicine, Harvard Medical School and Harvard Pilgrim Health Care Institute, Boston, MA. 3. Infection Control Department, Brigham and Women's Hospital, Boston, MA. 4. Society for Healthcare Epidemiology of America, Arlington, VA. 5. Division of Pulmonary and Critical Care Medicine, Rush University School of Medicine, Chicago, IL. 6. Critical Care Societies Collaborative-American Association of Critical-Care Nurses, American College of Chest Physicians, American Thoracic Society, Society of Critical Care Medicine. 7. School of Nursing, Critical and Acute Care, University of Virginia, Charlottesville, VA. 8. Department of Anesthesiology and Critical Care, Perelman School of Medicine at the University of Pennsylvania, Philadelphia, PA. 9. Division of Infectious Diseases, University of Iowa Carver College of Medicine, Iowa City, IA. 10. Healthcare Infection Control Practices Advisory Committee Surveillance Working Group, Atlanta, GA. 11. Infection Prevention and Control Department, Rochester General Health System, Rochester, NY. 12. Association for Professionals in Infection Control and Epidemiology, Washington, DC. 13. Department of Medicine, Medical College of Wisconsin, Milwaukee, WI. 14. Department of Cardiology, Zablocki VA Medical Center, Milwaukee, WI. 15. Hospital Epidemiology and Quality Improvement, The Clinical Center, National Institutes of Health, Bethesda, MD. 16. Department of Respiratory Care, Massachusetts General Hospital, Boston, MA. 17. Department of Anesthesia, Harvard Medical School, Boston, MA. 18. American Association for Respiratory Care, Irving, TX. 19. Division of Pulmonary and Critical Care Medicine, Tufts Medical Center, Boston, MA. 20. Division of Pulmonary and Critical Care Medicine, Washington University, St. Louis, MO. 21. Division of Pulmonary,
}

surveillance definitions and to propose new approaches to VAP surveillance in adult patients (Table). The charges to the Working Group were to:

1) Critically review a draft, streamlined VAP surveillance definition developed for use in adult patients;

Critical Care, and Sleep, Warren Alpert Medical School at Brown University, Rhode Island Hospital, Providence, RI. 22. Department of Internal Medicine, Texas A\&M Health Science Center, College Station, TX. 23. Infectious Diseases Society of America, Arlington, VA. 24. New York State Department of Health, Bureau of Healthcare-Associated Infections, Albany, NY. 25. Council of State and Territorial Epidemiologists, Atlanta, GA. 26. Office of Disease Prevention and Health Promotion, U.S. Department of Health and Human Services, Washington, DC. 27. Department of Surgery, Anesthesiology and Critical Care Medicine, Johns Hopkins University School of Medicine, Baltimore, MD.

Disclaimer: The findings and conclusions in this report are those of the authors and do not necessarily represent the official position of the Centers for Disease Control and Prevention, the American Association for Respiratory Care, the Association of Professionals in Infection Control and Epidemiology, the Council of State and Territorial Epidemiologists, or the Infectious Diseases Society of America.

This article is an executive summary of a report from the Centers for Disease Control and Prevention Ventilator-Associated Pneumonia Surveillance Definition Working Group published in Critical Care Medicine (Magill SS, Klompas M, Balk R, Burns SM, Deutschman CS, Diekema $\mathrm{D}$, et al. Developing a new, national approach to surveillance for ventilator-associated events. Crit Care Med 2013;41(11):2467-2475). The full report provides a comprehensive description of the Working Group process and outcome.

Financial support: The work described herein was supported by the Centers for Disease Control and Prevention (CDC). 
Table. VAP Surveillance Definition Working Group Organizations, Representatives, and Federal Participants

\begin{tabular}{ll}
\hline \hline \multicolumn{1}{c}{ Organization } & \multicolumn{1}{c}{ Representative(s) } \\
\hline American Association of Critical-Care Nurses & Suzanne Burns and Beth Hammer \\
American Association for Respiratory Care & Dean Hess \\
American College of Chest Physicians & Robert Balk and David Gutterman \\
American Thoracic Society & Nicholas Hill and Mitchell Levy \\
Association of Professionals in Infection Control and Epidemiology & Linda Greene \\
Council of State and Territorial Epidemiologists & Carole VanAntwerpen \\
Healthcare Infection Control Practices Advisory Committee & Daniel Diekema \\
$\quad$ Surveillance Working Group & Edward Septimus \\
Infectious Diseases Society of America & Michael Klompas \\
Society for Healthcare Epidemiology of America & Clifford Deutschman, Marin Kollef, and Pamela Lipsett \\
Society of Critical Care Medicine & Don Wright \\
United States Department of Health and Human Services, Office of & \\
$\quad$ Disease Prevention and Health Promotion & David Henderson \\
National Institutes of Health & Scott Fridkin, Alice Guh, Shelley Magill, Teresa Horan, others \\
Centers for Disease Control and Prevention, Division of Healthcare & \\
$\quad$ Quality Promotion & \\
\hline VAP $=$ ventilator-associated pneumonia & \\
\hline
\end{tabular}

DISCLOSURES: Dr Klompas received grant support from the CDC, U.S. Food and Drug Administration, and the Office of the National Coordinator for Health IT. Dr Balk received grant support from the CDC and bioMérieux for participation in the EPIC CAP study (CDC) and the Procalcitonin in ICU antibiotic stewardship study (CDC and bioMérieux). Dr Deutschman received grant support from the National Institute of General Medical Sciences. Dr Diekema received grant support from Merck, Cerexa, bioMériuex, PurThread Technologies, and Pfizer. Dr Klompas received support for travel from the CDC, Society of Healthcare Epidemiologists of America, Association of Professionals in Infection Control, and the Duke University Infection Control Outreach Network. Dr Balk received support for travel from the CDC and Critical Care Societies Collaborative. Dr Burns received support for travel from the American Association of Critical-Care Nurses (AACN; meeting unrelated to this study). Drs Deutschman and Lipsett received support for travel from the Society of Critical Care Medicine. Ms Greene received support for travel from the Department of Health and Human Services/CDC. Ms Greene consults for INC. Dr Hess consulted for Philips Respironics, ResMed, Pari, and Breathe, and received honorarium from Covidien and Maquet. Ms Greene lectured for Premier, Advanced Sterilization Products, and APIC. Ms Burns lectured for AACN (annual national conference). Ms Greene presented speeches for Covidien and Maquet. Dr Septimus received an honorarium for a lecture. Dr Klompas received support from the Society of Healthcare Epidemiologists of America for the development of educational presentations. Ms Greene is employed by the Rochester General Hospital. Dr Lipsett has board membership with the Society of Critical Care Medicine. Dr Deutschman received a stipend for his Presidency with the Society of Critical Care Medicine. Dr Deutschman received royalties from Elsevier for the textbook, Evidence-based Practice of Critical Care Medicine. Ms Burns receives royalties from McGraw-Hill for books endorsed by AACN. Ms Greene receives royalties from Up-To-Date, Jones and Bartlett, and McGraw-Hill. The remaining authors have disclosed that they do not have any potential conflicts of interest.

Correspondence: Shelley S Magill MD PhD, Division of Healthcare Quality Promotion, Centers for Disease Control and Prevention, 1600 Clifton Road, MS A-24, Atlanta GA 30329. Email: smagill@cdc.gov.

DOI: $10.4187 /$ respcare.02759
2) Suggest modifications to enhance the reliability and credibility of the surveillance definition within the critical care and infection prevention communities;

3) Propose a final adult surveillance definition algorithm, to be implemented in the CDC's National Healthcare Safety Network (NHSN), taking into consideration the potential future use of the definition algorithm in public reporting, interfacility comparisons, and pay-forreporting and pay-for-performance programs.

The Working Group's surveillance definition algorithm, which is referred to as the ventilator-associated events or VAE surveillance definition algorithm, represents a purposeful departure from VAP toward more general, objective measures of conditions and complications occurring in patients on mechanical ventilation (Figure; VAE surveillance protocol available at: http://www.cdc.gov/nhsn/ acute-care-hospital/vae/index.html). The VAE surveillance definition algorithm uses a tiered approach, moving from measures of ventilator-associated conditions (VAC), to infection-related ventilator-associated complications (IVAC), to possible and probable VAP.

The first tier of VAE surveillance, VAC, seeks to identify episodes of sustained respiratory deterioration, and will capture both infectious and non-infectious conditions and complications occurring in mechanically ventilated patients. VAC is defined by a sustained period of worsening oxygenation that immediately follows a baseline period of stability or improvement on the ventilator. To meet the VAC definition, a mechanically ventilated patient must have at least 2 calendar days of stable or decreasing daily minimum positive end-expiratory pressure (PEEP) or fraction of inspired oxygen $\left(\mathrm{F}_{\mathrm{IO}_{2}}\right)$, followed by at least 2 days 
Patient has a baseline period of stability or improvement on the ventilator, defined by $\geq 2$ calendar days of stable or decreasing daily minimum $\mathrm{FiO}_{2}$ or PEEP values. The baseline period is defined as the two calendar days immediately preceding the first day of increased daily minimum PEEP or $\mathrm{FiO}_{2}$.

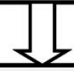

After a period of stability or improvement on the ventilator, the patient has at least one of the following indicators of worsening oxygenation 1) Minimum daily $\mathrm{FiO}_{2}$ values increase $\geq 0.20$ (20 points) over the daily minimum $\mathrm{FiO}_{2}$ in the preceding 2 calendar days (the baseline period), for $\geq$ 2 calendar days

2) Minimum daily PEEP values increase $\geq 3 \mathrm{cmH}_{2} \mathrm{O}$ over the daily minimum PEEP in the preceding 2 calendar days (the baseline period), for $\geq 2$ calendar days

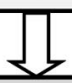

Ventilator-Associated Condition (VAC)

On or after calendar day 3 of mechanical ventilation and within 2 calendar days before or after the onset of worsening oxygenation, the patient meets both of the following criteria:

1) Temperature $>38^{\circ} \mathrm{C}$ or $<36^{\circ} \mathrm{C}$, OR white blood cell count $\geq 12,000$ cells $/ \mathrm{mm}^{3}$ or $\leq 4,000$ cells $/ \mathrm{mm}^{3}$ AND

2) A new antimicrobial agent(s)* is started, and is continued for $\geq 4$ calendar days

*See VAE surveillance protocol (available at: $h \mathrm{ttp}: / / w w w . c d c . g o v / n h s n / a c u t e-c a r e-h o s p i t a l / v a e / i n d e x . h t m l)$ for eligible agents

On or after calendar day 3 of mechanical ventilation and within 2 calendar days before or after the onset of worsening oxygenation, ONE of the following criteria is met:

1) Purulent respiratory secretions (from one or more specimen collections)

- Defined as secretions from the lungs, bronchi, or trachea that contain $\geq 25$ neutrophils and $\leq 10$ squamous epithelial cells per low power field [lpf, x100] (or corresponding semiquantitative results)

2) Positive culture (qualitative, semi-quantitative or quantitative) of sputum*, endotracheal aspirate*, bronchoalveolar lavage*, lung tissue, or protected specimen brushing*

*Excludes the following:

- Normal respiratory/oral flora, mixed respiratory/oral flora or equivalent

- Candida species or yeast not otherwise specified

- Coagulase-negative Staphylococcus species

- Enterococcus species

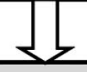

Infection-related Ventilator-Associated Complication (IVAC)

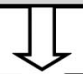

On or after calendar day 3 of mechanical ventilation and within 2 calendar days before or after the onset of worsening oxygenation, ONE of the following criteria is met:

1) Purulent respiratory secretions (from one or more specimen collections-and defined as for possible VAP)

AND one of the following:

- Positive culture of endotracheal aspirate*, $\geq 10^{5} \mathrm{CFU} / \mathrm{ml}$ or equivalent semi-quantitative result

- Positive culture of bronchoalveolar lavage*, $\geq 10^{4} \mathrm{CFU} / \mathrm{ml}$ or equivalent semi-quantitative result

- Positive culture of lung tissue, $\geq 10^{4} \mathrm{CFU} / \mathrm{g}$ or equivalent semi-quantitative result

- Positive culture of protected specimen brush*, $\geq 10^{3}$ $\mathrm{CFU} / \mathrm{ml}$ or equivalent semi-quantitative result

*Same organism exclusions as noted for Possible VAP.

2) One of the following (without requirement for purulent respiratory secretions):

- Positive pleural fluid culture (where specimen was obtained during thoracentesis or initial placement of chest tube and NOT from an indwelling chest tube)

- Positive lung histopathology

- Positive diagnostic test for Legionella spp.

- Positive diagnostic test on respiratory secretions for influenza virus, respiratory syncytial virus, adenovirus, parainfluenza virus, rhinovirus, human metapneumovirus, coronavirus

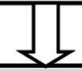

Probable Ventilator-Associated Pneumonia

Figure. Ventilator-associated events surveillance definition algorithm. Available at: http://www.cdc.gov/nhsn/acute-care-hospital/vae/ index.html. PEEP = positive end-expiratory pressure. $\mathrm{F}_{\mathrm{IO}_{2}}=$ fraction of inspired oxygen. $\mathrm{VAP}=$ ventilator-associated pneumonia. $\mathrm{CFU}=$ colony-forming unit. 
of increased daily minimum PEEP or $\mathrm{F}_{\mathrm{IO}_{2}}$, where the increase in the daily minimum PEEP is $\geq 3 \mathrm{~cm} \mathrm{H}_{2} \mathrm{O}$ greater than the daily minimum PEEP during the baseline period, or where the increase in the daily minimum $\mathrm{F}_{\mathrm{IO}_{2}}$ is $\geq 0.20$ (or 20 percentage points in oxygen concentration) greater than the daily minimum $\mathrm{F}_{\mathrm{IO}_{2}}$ during the baseline period. For example, if a patient's daily minimum $\mathrm{F}_{\mathrm{IO}_{2}}$ requirement on days 4 and 5 of mechanical ventilation is 0.40 , then the patient's daily minimum $\mathrm{F}_{\mathrm{IO}_{2}}$ requirement would need to be at least 0.60 on days 6 and 7 of mechanical ventilation for the VAC definition to be met. The Working Group's decisions to set specific thresholds of $3 \mathrm{~cm} \mathrm{H}_{2} \mathrm{O}$ and 0.20 (20 points) for the increases in PEEP and $\mathrm{F}_{\mathrm{IO}_{2}}$, respectively, and to define a "sustained" increase as an increase persisting for at least 2 calendar days, were based on expert opinion of what criteria would likely identify clinically important events, while minimizing inadvertent inclusion of other types of events resulting in transient changes in oxygenation - such as surgery or performance of other procedures. Thresholds were also selected based upon published data indicating that increases of $\geq 2.5 \mathrm{~cm} \mathrm{H} \mathrm{H}_{2} \mathrm{O}$ in PEEP or $\geq 0.15$ (15 points) in $\mathrm{F}_{\mathrm{IO}_{2}}$ sustained for at least 2 days were associated with longer duration of mechanical ventilation, ICU and hospital stays, and increased mortality. ${ }^{1}$ Subsequently, additional data have been published that support the Working Group's approach to VAC. ${ }^{2}$

The second tier, IVAC, attempts to identify the subset of VACs that are potentially related to infection, as evidenced by an abnormal white blood cell count or temperature and initiation of a new antimicrobial agent. IVAC will likely capture patients with pulmonary infections and extrapulmonary infections of sufficient severity to trigger respiratory deterioration. The Working Group recognized the low predictive value of an abnormal temperature or white blood cell count in ICU patients, and Klompas and colleagues have shown that the addition of fever or abnormal white blood cell count to VAC definition does not substantially enhance the definition's predictive value for death. ${ }^{2}$ Nevertheless, these are objective and readily available signs that are frequently used at the bedside to assess for the presence of infection. The additional required criterion of starting a new antimicrobial, where the new agent is continued for at least 4 days, may add specificity and clinical credibility to the IVAC definition, although data are needed.

The third tier, possible and probable VAP, attempts to zero in on the subset of IVAC patients with respiratory infections as manifested by objective evidence of purulent respiratory secretions (where purulence is defined using quantitative or semiquantitative criteria for the number of neutrophils on Gram-stain) and/or positive results of microbiological tests performed on respiratory tract specimens. The possible VAP definition is met with the pres- ence of purulent secretions or a positive lower respiratory tract culture (showing any growth); the probable VAP definition requires purulent secretions in addition to a positive lower respiratory tract culture meeting certain quantitative or semiquantitative thresholds of pathogen growth. Organisms that are uncommonly regarded as true VAP pathogens are excluded from possible and probable VAP culture criteria (with the exception of lung tissue cultures): Candida spp., coagulase-negative staphylococci, and Enterococcus spp. The probable VAP definition can also be met based upon the presence of a positive pleural fluid culture, lung tissue with histopathological evidence of infection, or positive diagnostic tests for Legionella or selected respiratory tract viruses, without the concomitant requirement for purulent secretions. Although data have shown that requiring purulent secretions or positive cultures in patients who have met a VAC definition actually diminishes the association between mortality and $\mathrm{VAC},{ }^{2}$ the Working Group felt that it was important to provide definitions within the VAE algorithm that more closely resemble VAP diagnostic criteria used at the bedside.

This tiered approach is believed to be the most appropriate approach in the current environment. It acknowledges limitations in the ability to accurately identify VAP for surveillance purposes-simply labeling an event "VAP" does not make it so-and focuses instead on a more general measure of complications of mechanical ventilation. This approach may also reduce the likelihood of definition gaming or manipulation that could artificially lower event rates.

Two features of the VAE surveillance definition algorithm are of particular note. First, radiographic evidence of pneumonia is not included as a criterion in any tier of the algorithm because of lack of specificity, and the subjectivity inherent in facilities' and individual providers' practices in ordering, performing, interpreting and reporting results of chest radiographs. Second, only VAC and IVAC (and therefore the overall VAE rate-the rate of all events meeting at least the VAC definition-and the rate of all events meeting at least the IVAC definition) are intended to be possible candidates for future use in public reporting, interfacility comparisons, and pay-for-performance programs. The VAC and IVAC definitions use criteria based on data anticipated to be available from most mechanically ventilated patients and less subject to manipulation or gaming. By contrast, the third definition tier, possible and probable VAP, was developed to be used only in internal quality improvement. These VAP definitions include criteria based on documentation of purulent secretions and/or microbiological findings and are more in keeping with traditional clinical constructs of VAP. Because of the substantial variability in the ordering and collection of lower respiratory tract specimens, and in laboratory processing of specimens and reporting of results, the Working Group 
determined that it was not appropriate to include these data elements in the VAC and IVAC definitions.

An iterative process for refining the definitions must be ensured as experience using the definition algorithm accumulates in the coming years. Although there was clearly a need to establish a new surveillance approach in the NHSN, there is also an urgent need to advance the science of surveillance for VAP and other VAE. The VAE surveillance definition algorithm should be studied, validated, and improved in an ongoing manner. Evaluation and refinement of the definitions should be conducted in collaboration with members of the Working Group and other members of stakeholder communities and organizations. The Working Group is already discussing potential modifications based on user feedback received during the first 3 months of VAE surveillance.

The VAP Surveillance Definition Working Group's new approach to surveillance in mechanically ventilated adults acknowledges the current limitations in VAP diagnosis and the potential benefit in focusing surveillance on an objective, reliable, but more general measure of significant conditions and complications that occur in patients on ventilators. VAE surveillance was implemented by the CDC's NHSN in January 2013. While much work remains, we believe this innovative approach to surveillance has significant potential to increase the validity of comparisons among healthcare facilities and, more importantly, to improve measurement and patient safety in the ICU.

\section{REFERENCES}

1. Klompas M, Khan Y, Kleinman K, Evans RS, Lloyd JF, Stevenson $\mathrm{K}$, et al. Multicenter evaluation of a novel surveillance paradigm for complications of mechanical ventilation. PLoS One 2011;6:e18062.

2. Klompas M, Magill S, Robicsek A, Strymish JM, Kleinman K, Evans RS, et al. Objective definitions for ventilator-associated pneumonia. Crit Care Med 2012;40:3154-3161. 